Gut, 1977, 18, 515-523

\title{
Effects of proximal gastric vagotomy (PGV) followed by total vagotomy (TV) on postprandial and fasting myoelectrical activity of the canine stomach and duodenum ${ }^{1}$
}

\author{
P. AEBERHARD ${ }^{2}$ AND B. S. BEDI
}

From the Department of Surgery, Western Infirmary and the Wellcome Surgical Research Institute, Glasgow

SUMMARY Experiments were designed to study the effect of proximal gastric vagotomy (PGV) followed by total vagotomy (TV) on the myoelectrical activity of the canine stomach and duodenum after a meat meal and during fasting. Dogs were prepared with chronically implanted $\mathrm{Ag}-\mathrm{AgCl}-$ electrodes on the stomach and duodenum. Recordings of electrical activity were made for one hour after the ingestion of a meat meal and records of four to six hours were taken on the fasting animals. In the postprandial records no effect on co-ordination of antral and duodenal myoelectrical activity was found after either PGV or TV. These results suggest that the co-ordinating mechanism is not dependent on vagal innervation. Impairment of gastric emptying after TV does not appear to be due to disturbance of antral-duodenal conduction. Normal patterns of fasting activity were found in control and after PGV. They were grossly disturbed after TV, probably because of delayed gastric emptying. These findings suggest that PGV does not delay gastric emptying as does TV, and that the proximal vagal supply to the stomach is unimportant in the control of fasting activity.

During the emptying of a meal the motor activity of the duodenum undergoes cyclic changes in rhythm with the gastric antrum, suggesting the presence of a co-ordinating mechanism. According to Thomas (1957), Joseph and Meltzer first described the antralduodenal relationship in 1910, and the concept was further elaborated by Thomas and Crider (1935). More detailed knowledge of the underlying mechanisms has been obtained by the use of electromyography. Records of gastric and duodenal activity show an abrupt change of the pattern at the gastroduodenal junction. Electrodes placed $1 \mathrm{~cm}$ apart on both sides of the junction will record completely different types of activity. The frequency of the basic electrical rhythm (BER) in the dog stomach is four to five per minute, whereas the frequency in the duodenum is 16 to 20 per minute.

\footnotetext{
${ }^{1}$ This work was supported in part by the Swiss Stiftung für biologisch-medizinische Stipendien and the Clark-Joller Fonds.

${ }^{2}$ Address for correspondence: Dr. P. Aeberhard, University Department of Surgery, Inselspital, CH-3010 Berne, Switzerland.

Received for publication 13 December 1976
}

Allen et al., (1964) studied the myoelectrical activity of the stomach and duodenum in the conscious dog with chronically implanted electrodes. Whereas BER of the stomach and duodenum were found to be unrelated in either fasted or fed dogs, the timing of occurrence of duodenal action potentials (AP) was shown to be related to the antral pacesetter potentials (PSP) in the postprandial state.

Co-ordination of action potentials is lost after pyloric trans-section and restoration of the continuity by end-to-end anastomosis of the mucosa only (Bedi and Code, 1972). It has been concluded from this finding that the correlation of the electrical activities of antrum and duodenum is accomplished by messages via the neural and muscular elements in the wall of the gastroduodenal junction rather than by the passage of chyme from the stomach to the duodenum.

Fasting myoelectrical activity of the canine stomach and small bowel is organised into a cyclic pattern which is detectable as long as the animal is fasting but will immediately be interrupted and replaced by the postprandial pattern of activity upon feeding. This 'interdigestive myoelectrical complex' 
(IDMEC) was first described by Szurszewski (1969) in the small bowel of the fasting dog with chronically implanted electrodes. The complex consists of a caudad moving band of large-amplitude action potentials, originating simultaneously in the stomach and duodenum (Code and Marlett, 1975) and migrating distally over the entire small bowel in recurring cycles every 80 to 180 minutes. Khan and Bedi (1972) found that, in dogs after bilateral transthoracic vagotomy, the recurring complexes in the stomach were completely disrupted, suggesting vagal control of the IDMEC. More recent work on the canine stomach and small bowel by Marik and Code (1975) and on the small bowel by Weisbrodt et al., (1975) has shown that the complexes still occur after total vagotomy but may be modified by the absence of vagal innervation.

The work presented here was undertaken further to elucidate the role of vagal innervation in control of gastric and duodenal myoelectrical activity by studying the effects of proximal gastric vagotomy followed by total vagotomy (1) on the antral-duodenal co-ordination of action potentials in the postprandial state; and (2) on the interdigestive myoelectrical complex.

\section{Methods}

ANIMALS AND SURGICAL PROCEDURES

Four mongrel dogs ranging in weight from 18 to $25 \mathrm{~kg}$ were studied. All observations were made in the fully conscious animals trained to rest quietly in a Pavlov type of stand, and supported comfortably in canvas slings.

IMPLANTATION OF ELECTRODES

Six silver-silver chloride electrodes were implanted to detect the electrical activities of the gastric antrum and duodenum. Each electrode was $0.6 \mathrm{~mm}$ in diameter and projected $2 \mathrm{~mm}$ from a disk of dental acrylic. Two holes and a notch were made in the disk to make it easier to fix it to the wall of the gut. Each electrode was soldered, within the acrylic disk, to a PVC-insulated copper wire which served as a lead from the electrode to a junction implanted in the anterior abdominal wall. The junction consisted of a standard miniature seven-pin radio tube socket mounted with dental acrylic in a stainless steel cannula.

With the dogs under general anaesthesia and with the usual aseptic surgical techniques, the acrylic plates with electrodes were sutured to the serosal surface of the ventral aspect of the antrum and duodenum. The gastroduodenal junction was used as a reference point, and the distance and position of the electrodes are shown in Fig. 1. The stainless steel cannula containing the junction in the anterior abdominal wall was used as a reference electrode for monopolar recording.

\section{Proximal gastric vagotomy}

Proximal gastric vagotomy (PGV) was carried out as described by Wilbur and Kelly (1973). In order to check for preservation of antral vagal fibres after PGV, records of antral electrical activity after the intravenous injection of $\mathbf{0 . 1 5}$ to $\mathbf{0 . 2}$ units $/ \mathbf{k g}$ of regular insulin were taken on at least two occasions in each of the animals. The insulin was given during phase I of the interdigestive myoelectrical complex, in which spontaneously occurring action potentials are virtually absent and insulin-stimulated activity can be readily detected. In each of the tests the

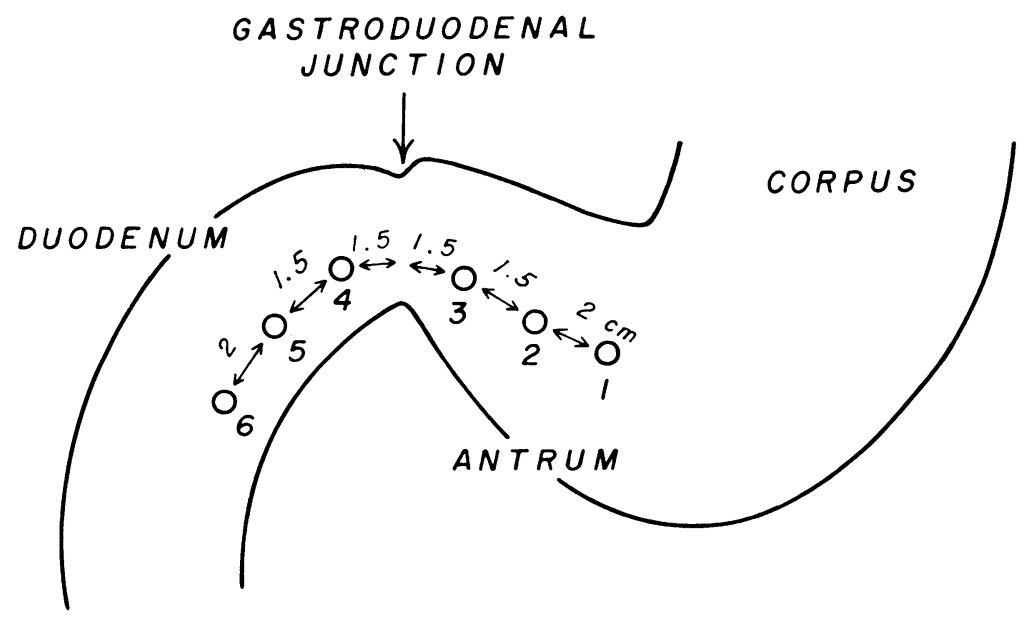

Fig. 1 Position of electrodes on antrum and duodenum. 
preservation of antral vagal innervation was demonstrated by the appearance, within 15 minutes of administration of the drug, of action potentials which lasted throughout the one hour recording period.

\section{Transthoracic vagotomy (TV)}

A thoracotomy in the left 8th intercostal space was carried out and, after exposure to an amount sufficient to display the union of branches from the left and right vagus nerves to form the anterior and posterior vagal trunks, $2 \mathrm{~cm}$ segments were resected from both vagal trunks. The ends of the nerves were ligated with 3-0 silk.

\section{Gastric fistulas and insulin tests}

Gastric cannulas of the Gregory type were inserted. After a recovery period of 10 to 12 days, insulin tests for completeness of secretory vagal denervation were performed. As at this time some gastric retention was still found in the dogs, the gastric fistulas were left open for at least $\mathbf{3 0}$ minutes before beginning collection of basal secretion. Basal secretion was collected in two 30 minute periods, and regular insulin was then injected intravenously at a dose of 0.15 to 0.2 units $/ \mathrm{kg}$ body weight. Secretion was collected in four 30 minute portions over the following two hours. At 30 minutes after the injection of insulin, a blood sample was taken for blood sugar determination. Gastric acid was determined from $1 \mathrm{ml}$ aliquots by titration with $\mathrm{N} / 100 \mathrm{NaOH}$, using phenol red as an indicator. The result was interpreted using Hollander's criterion of a rise in acid concentration by at least $20 \mathrm{~m} \mathrm{Eq} / 1$ over basal acid concentration in any postinsulin sample or a rise of at least $10 \mathrm{~m} \mathrm{Eq} / 1$ when the basal secretion was anacid (Hollander, 1948). Total acid output over the two postinsulin hours was determined as well. Two insulin tests were performed in each dog. In each of the three dogs studied after TV the insulin tests were negative according to Hollander's criterion. Blood sugar levels in blood samples taken at 30 minutes after insulin were below $40 \mathrm{mg} / \mathrm{dl}$ in all the experiments.

\section{EXPERIMENTAL DESIGN AND RECORDING METHODS}

Recording of electrical activity was started two weeks after implantation of electrodes. The animals were fasted for at least 18 hours before each recording session. Electrical activity was recorded in the fasting animal and after feeding a can of meat. Records of four to six hours' duration were taken during fasting, and for one hour after feeding. A permanent record of the changes in potential was obtained on an eight-channel rectilinear ink-jet writer (Elema-Schönander Mingograph EEG 8). Amplifiers with flat frequency response up to $15 \mathrm{~Hz}$ and time constants of $0 \cdot 15$ and $0 \cdot 3 \mathrm{~s}$ were used.

After an initial series of observations was completed proximal gastric vagotomy (PGV) was performed. The animals were allowed two weeks to recover, after which recording was resumed, and studies identical with those done before vagotomy were repeated during the following six weeks. In one dog, infection developed around the cannula and electrode system, and this animal could not be used for further study. The remaining three dogs underwent a third operation eight weeks after PGV when transthoracic vagotomy (TV) was performed. The animals were able to take their normal kennel diet within $\mathbf{1 0}$ days of TV. One animal retained its pre-vagotomy weight, one gained $2 \mathrm{~kg}$, and the third lost $1 \frac{1}{2} \mathrm{~kg}$ in the three months after TV. Recording of electrical activity was resumed four weeks after TV. Three animals underwent the whole experimental programme, which consisted in at least three records of fasting and postprandial activity during the control period, after PGV and after TV. One animal underwent study of postprandial activity during the control period and after PGV only.

Three months after TV the animals were taken back to theatre for insertion of gastric cannulas. Thereafter, insulin tests for completeness of vagotomy were performed.

\section{ANALYSIS OF RECORDS}

For analysis of the electromyograms the records from one electrode site each on the stomach and duodenum were used.

\section{POSTPRANDIAL ACTIVITY}

This was studied by a method of cross-correlation as used by Allen et al. (1964) and by Bedi and Code (1972). The objective of the method is to determine whether a particular event in one rhythmic sequence is related to a particular event in another cyclically recurring phenomenon. A consistently well-defined component of one of the recurring sequences is selected as a reference point, and its incidence of occurrence is determined within equal phases or equal temporal subdivisions of a cycle of the other recurring phenomenon. In this way, a phase-density distribution is compiled for the occurrence of one series of events during the cycles of the other. Any constant temporal correlation between the two series of events will appear as clusterings in certain phases and this should persist in successive samples and experiments.

The maximal negative deflection was selected as 


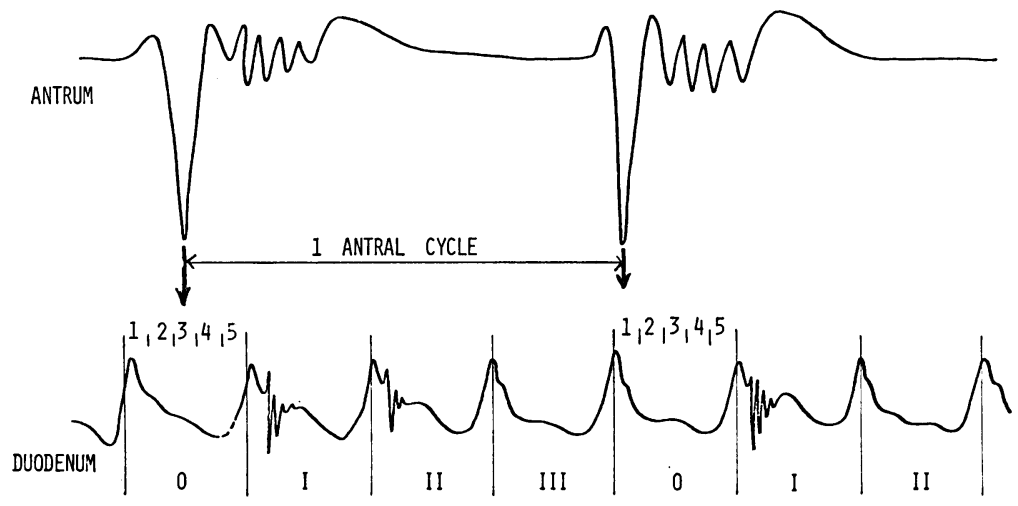

Fig. 2 The method of crosscorrelation analysis. Simultaneous recording of electrical activity from antrum and duodenum. Further explanation in the text. the fixed point of reference in each cycle of the antral PSP, and the maximal positive deflection was selected as the point of reference in each cycle of the duodenal PSP. Four complete duodenal cycles usually occurred during each antral cycle.

For subsequent identification, the duodenal cycle during which an antral cycle started was designated as 'cycle 0', and those occurring during the remainder of the antral cycle were numbered in sequence, I, II, III, and occasionally IV (Fig. 2).

To cross-correlate antral with duodenal activity, each duodenal cycle 0 was divided into five equal temporal segments, and the fifth, into which the onset of the antral PSP fell, was determined, a special triangulated, clear plastic cursor being used to facilitate the determination.

As the first step in analysis of the records, all duodenal PSP cycles were identified as cycle $0, I$, II, III, or IV. Then, the occurrence of APs in each cycle was noted. Next, the fifth into which the antral PSP fell was noted for each duodenal cycle 0 , and finally the incidence of APs in duodenal cycles 0 , I, II, III was tabulated according to the fifth of the duodenal cycle 0 into which the antral reference point fell. A period of 200 consecutive antral cycles was analysed in each experiment and the data of each experiment were arranged in a table.

The incidence of antral PSPs in each fifth of the duodenal cycle 0 was assessed statistically by using a chi square test (Freund, 1973) on the expectancy of equal incidence in each fifth.

For the study of the relation between the antral BER and the occurrence of APs in the duodenum, each cell in the table of incidence of APs in duodenal cycles $\mathbf{0}$, I, II, and III was assigned its position on a time scale extending from the antral reference point taken as zero time and graduated in fifths of a duodenal cycle. The temporal distribution of duodenal APs in relation to the antral BER was then represented graphically as a histogram. The histograms were generated by a Hewlett-Packard 9100 B calculator and 9125 A calculator plotter.

\section{Analysis of fasting records}

When there was a clearly recognisable cyclic pattern, cycle length and duration of the phases of the interdigestive myoelectrical complex were determined by direct inspection of the records. As the onset of phase I is particularly well defined in most of the records, this was taken as the point from which to measure cycle length. In part of the experiments a more detailed analysis was carried out by establishing the percentage of PSPs with APs per 100 seconds at one electrode site each in the stomach and duodenum throughout the time of recording. For diagrammatic representation of the results a simplified procedure similar to that used by Marik and Code (1975) has been adopted in which 0 to $5 \%$ incidence of action potentials were graphed as $\mathbf{0}$ or a horizontal line, 5 to $90 \%$ incidence as open rectangles, and the activity front of the complex with $90-100 \%$ incidence of APs was plotted as a solid rectangle.

In some of the records after TV, the variable configuration of the antral PSPs made the identification of APs rather difficult. In these cases the analysis was restricted to the study of the duodenal tracing.

\section{Results}

POSTPRANDIAL ACTIVITY

\section{Relationship between BER of antrum and duodenum} The incidence of onset of the antral PSP in each fifth of the 0 duodenal cycle was nearly equal. The $P$ value of the chi square test was $>0.9995$. Thus, previously reported findings that the BER of stomach and duodenum are unrelated were confirmed by our study. 


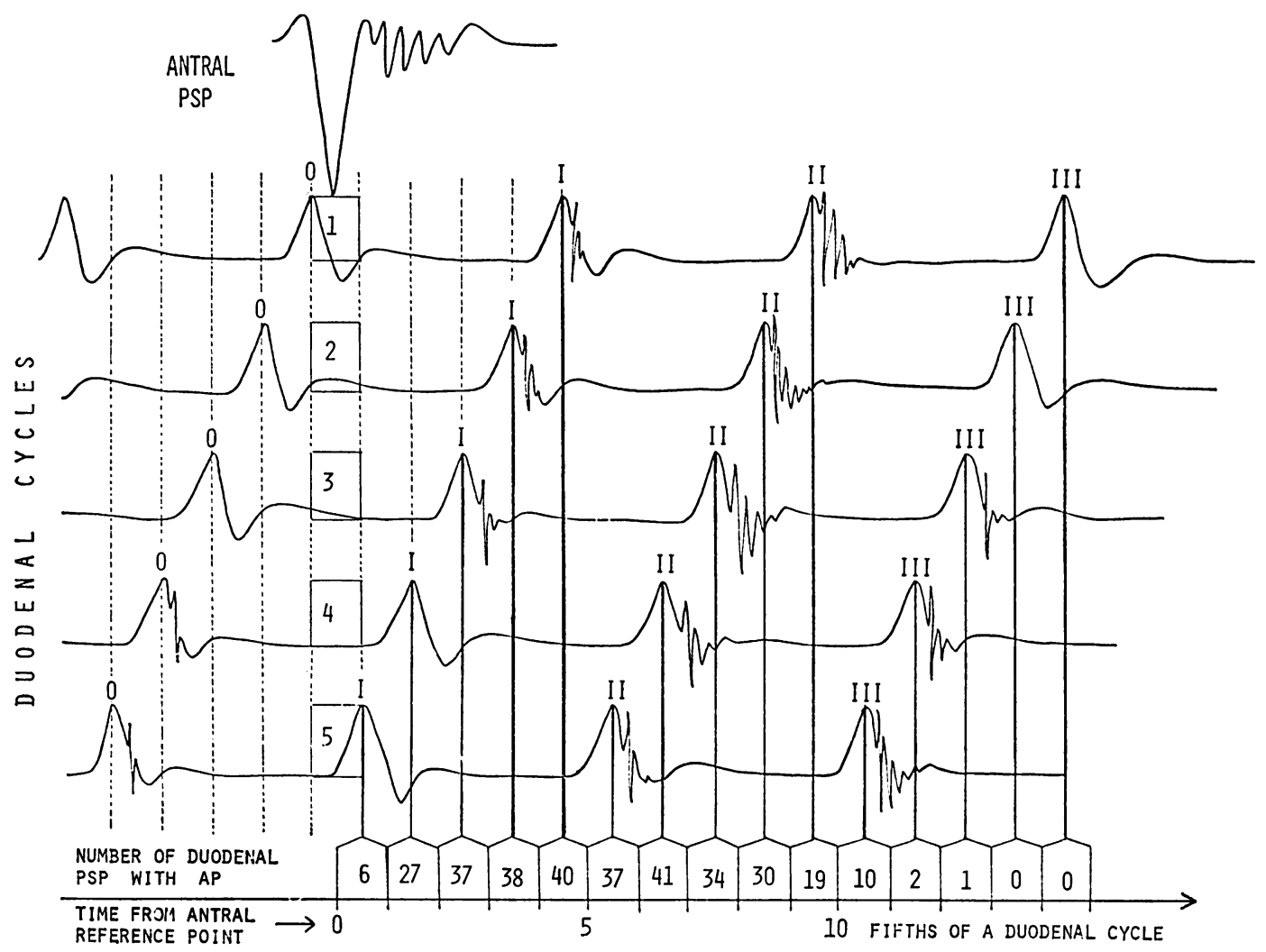

Fig. 3 How the data in Table 1 can be aligned on a time axis extending from the antral reference point to represent the temporal distribution pattern of duodenal APs in relation to the antral BER.

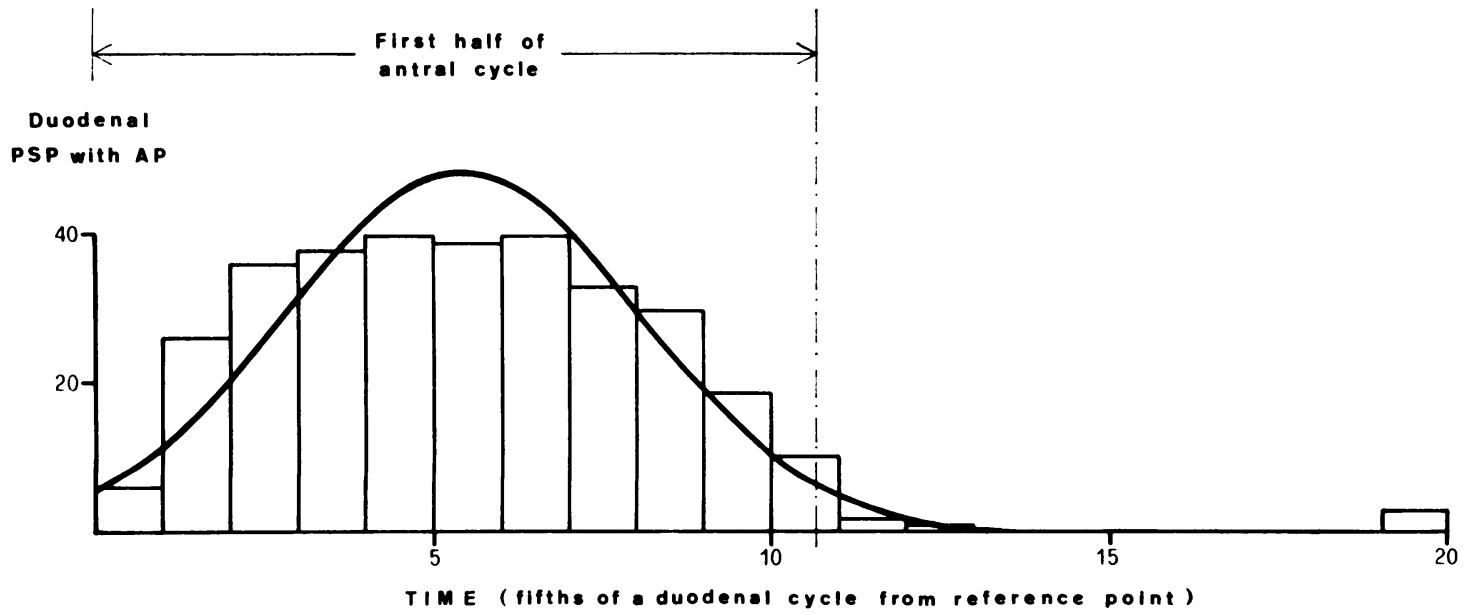

Fig. 4 Histogram of data from Table 1 and Fig. 3, with normal curve overlay: The majority of duodenal APs occur during the first half of the antral cycle. Frequency of antral BER was 4.4/min and duodenal frequency was $19 /$ min in this experiment. 
Relationship between incidence of duodenal action potentials and antral $B E R$

Results from the control period show that the incidence of action potentials with duodenal PSPs is temporally related to the antral BER. The relationship can be demonstrated in the form of a time-density distribution of occurrence of duodenal APs in relation to the antral BER. The probability for action potentials to occur in the duodenal bulb reaches its maximum during the first half of the

Table 1

\begin{tabular}{llllll}
\hline $\begin{array}{l}\text { Fifth of duo- } \\
\text { denal cycle } O \\
\text { into which the } \\
\text { antral PSP } \\
\text { falls }\end{array}$ & $\begin{array}{l}\text { Number of PSPs with APs in } \\
\text { duodenal cycles }\end{array}$ & cycle $O$ & cycle I & cycle II & \multicolumn{1}{l}{$\begin{array}{l}\text { Number of } \\
\text { antral PSPs }\end{array}$} \\
\hline 1st & 3 & 40 & 19 & 0 & \\
2nd & 0 & 38 & 30 & 0 & 40 \\
3rd & 0 & 37 & 34 & 1 & 41 \\
4th & 0 & 27 & 41 & 2 & 41 \\
5th & 0 & 6 & 37 & 10 & 38 \\
\hline
\end{tabular}

Total antral PSPs: 200. O: $\operatorname{dog}$ O.

antral cycle. This is demonstrated in Table 1 which shows the data of one experiment in dog O. Figure 3 illustrates how each cell of Table 1 is assigned its position on the time axis for calculation of the temporal distribution pattern of duodenal APs, and Fig. 4 shows the histogram with normal curve overlay drawn from the same data.

After proximal gastric vagotomy there was no change from the distribution pattern of duodenal AP's found in the control period.
After truncal vagotomy a pattern of regular postprandial activity was not always obtained after feeding $500 \mathrm{~g}$ meat. Meals of $1 \mathrm{~kg}$ were therefore given, which proved to be an adequate stimulus in every experiment. When recording of postprandial electrical activity was resumed at four weeks after truncal vagotomy, co-ordination could be demonstrated in the same way as before in two out of three dogs. In the third dog the distribution curve of duodenal APs was much more spread out throughout the antral cycles in the first two experiments, suggesting poor co-ordination, but when the studies were repeated on a weekly schedule, the previously established pattern reappeared and was then found consistently in three successive experiments.

In order to be able to compare the results from the control period statistically with those after PGV and after TV, a parameter is required which can be used as a measure for the degree of co-ordination.

The standard deviation of the duodenal APdistribution serves this purpose quite well: when the relationship between the antral BER and the timing of duodenal APs is strong, the scatter of duodenal APs around the maximum of their distribution curve will be quite small, whereas a weakening of the relationship will show as a widening of the duodenal AP-distribution curve. A lesser degree of co-ordination after vagotomy will be expected to result in an increase of the standard deviation of the duodenal AP-distribution pattern over that found in the control period. It was therefore thought that an

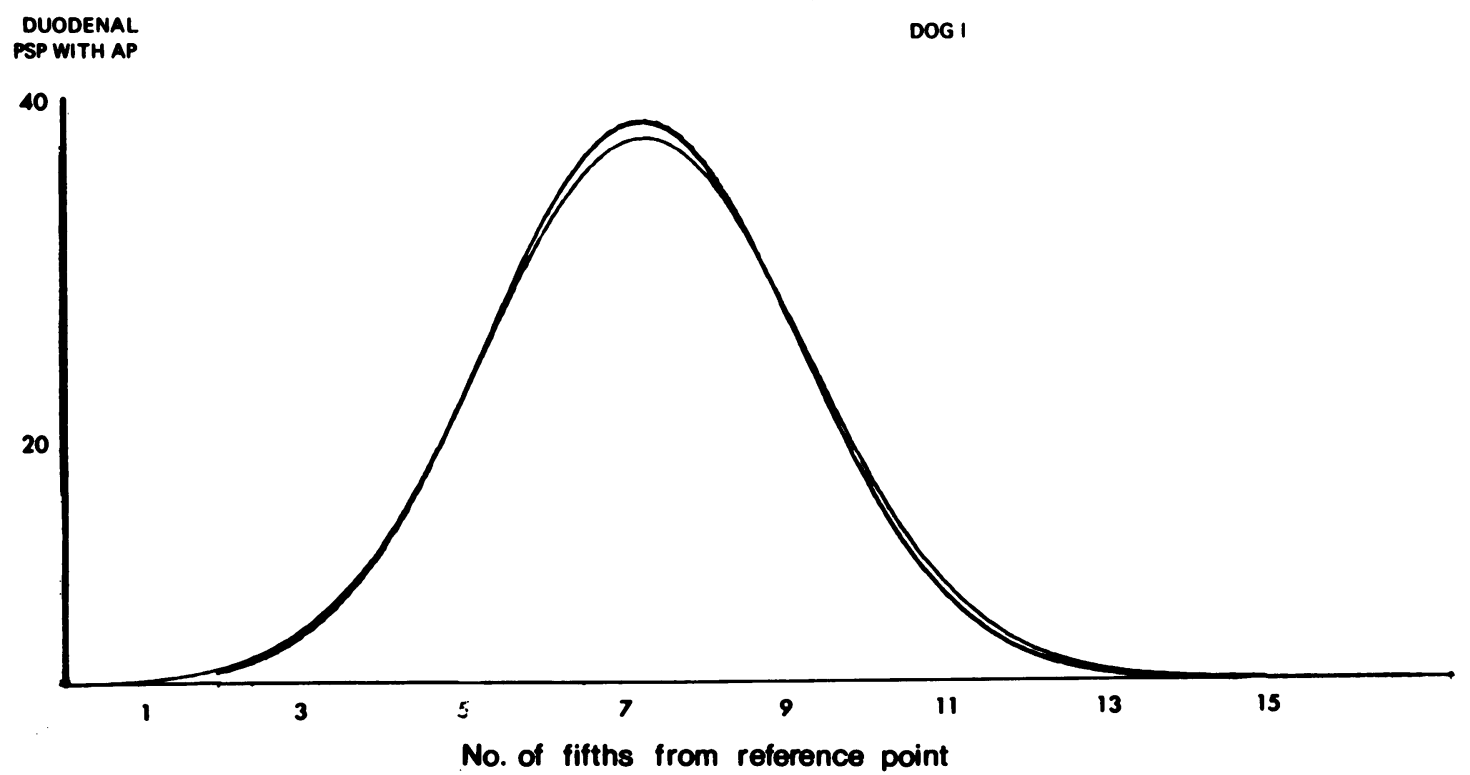

Fig. 5 Co-ordination of electrical activity across the gastroduodenal junction. 
analysis of variance of the standard deviations would show whether or not there was a significant difference in the degree of co-ordination between the results from the control period, after PGV, and after TV. This was done for the distribution patterns of all experiments in the three dogs which had gone through the complete experimental programme. No significant difference was found at the $1 \%$ level of significance.

This result is illustrated by Fig. 5 in which the normal curve overlays of one test each in the control period, after PGV, and after TV in dog I are superimposed on the same graph.

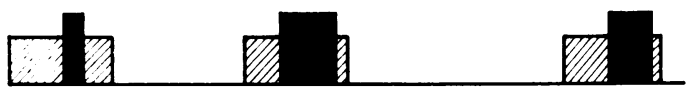

Stomach

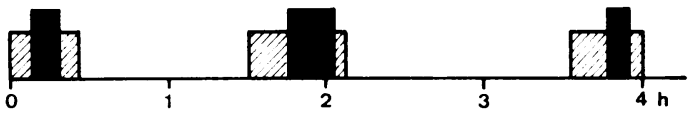

Duodenum
FASTING ACTIVITY

In the control period the IDMEC was readily detected in all three animals. PGV left the fasting pattern undisturbed. There was no effect on cycle length nor on the variability of the phases of the IDMEC (Table 2 and Figs. 6, 7). After TV the fasting

Table 2

\begin{tabular}{llll}
\hline & $\begin{array}{l}\text { Number of } \\
\text { cycles }\end{array}$ & Cycle length (min) & $\begin{array}{l}\text { Duration of phase } \\
I I-I V \text { (min) }\end{array}$ \\
\hline Control & 15 & $109 \cdot 5 \pm 16.4$ (S.D.) & $36.3 \pm 21.4$ (S.D.) \\
After PGV & 14 & $109 \pm 14$ & $42.6 \pm 8.6$ \\
\hline
\end{tabular}

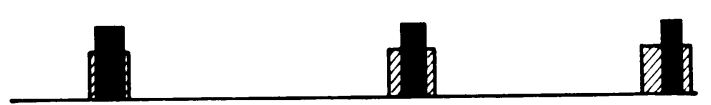

Stomach

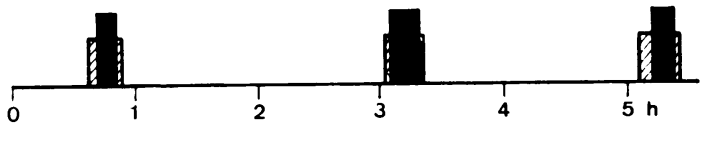

Duodenum

Fig. 6 The fasting pattern in the control period. (left) dog M, (right) dog I. In this Figure and Figs. 7, 8, and 9, 0 to $5 \%$ occurrence of APs is graphed as a horizontal line, 5 to $90 \%$ incidence as crosshatched rectangles, and 90 to $100 \%$ incidence as a solid black rectangle.

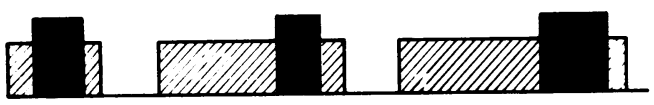

Stomach

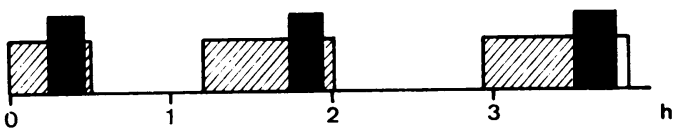

Duodenum

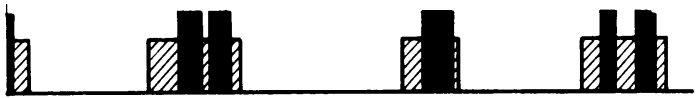

Stomach

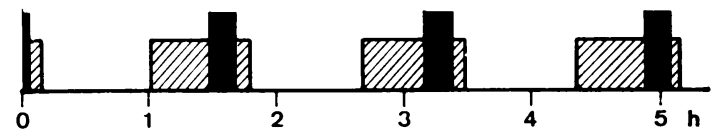

Duodenum

IDMEC cycles were registered in the duodenum (right). dog I, 44 days after TV. 
pattern was found to be grossly disturbed. The cyclic pattern seemed to be replaced by or submerged in a more continuous type of activity, with activity fronts appearing only occasionally on the tracings (Figs. $8,9)$. Occasionally the record could be interpreted to represent a delayed transition from the postprandial to the fasting pattern (Fig. 9a). On repeat tests there was no tendency for the normal fasting pattern to be restored during the observation period from one to three months after TV.
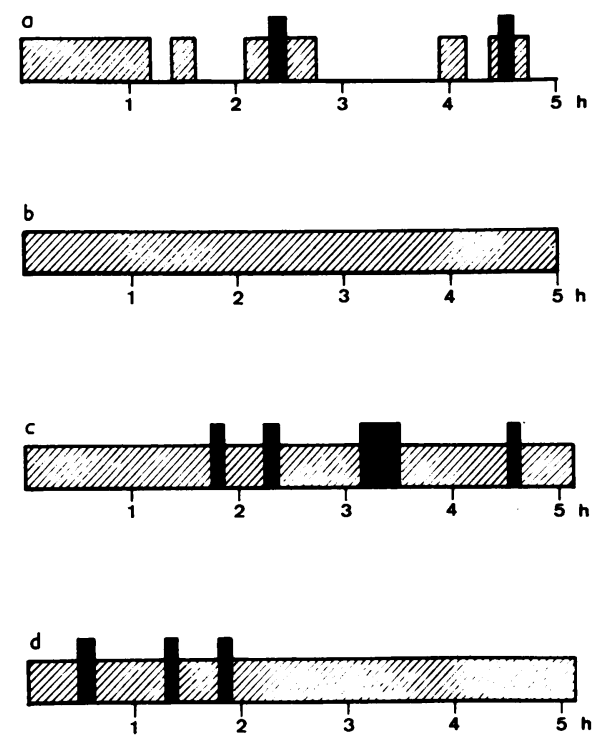

Fig. 9 Duodenal fasting activity after TV. (a) $\operatorname{dog} M$, 58 days after $T V$. This tracing probably represents a delayed transition from the postprandial to the fasting pattern. (b) dog $M, 79$ days after $T V$. (c) dog O, 42 days after $T V .(d)$ dog $O, 81$ days after $T V$.

\section{Discussion}

\section{POSTPRANDIAL ACTIVITY}

Co-ordination of postprandial electrical activity across the gastroduodenal junction was found to be well preserved after PGV in this study. The results after TV demonstrate the importance of a prolonged period of observation and repeat studies for assessment of the chronic effects of total vagotomy. One month after TV, co-ordinated postprandial activity was present in two of the three dogs. The third dog clearly showed restitution of previously impaired coordination during the second month after TV.

In our work we describe the events in the first hour after feeding. There is some evidence from previously published work (Stoddard and Duthie, 1973) that antral-duodenal co-ordination may be impaired by TV later in the postprandial stage.
The results of this study suggest that the coordinating mechanism is capable of functioning independently of the central connections of the vagus nerves. Impairment of gastric emptying after TV does not therefore appear to be due to disturbance of antral-duodenal electrical conduction.

FASTING ACTIVITY

In dogs studied between 18 and 24 hours after feeding, normal fasting patterns of myoelectrical activity are seen in control and after PGV. They are grossly disturbed in dogs studied one to three months after TV. Khan and Bedi (1972) reported total disruption of the interdigestive pattern after TV and pyloroplasty in the dog. In the study of Weisbrodt et al. (1975) in which no significant effect of TV on small bowel myoelectrical complexes was noted, the stomachs of the postvagotomy dogs were washed out via the gastric cannula three to four hours before recording, as it was noted that the stomach still contained food residue. Marik and Code (1975) similarly washed the stomachs out through an orogastric tube and fasted the animals for another 24 hours, when no interdigestive pattern of electrical activity had been obtained after a 24 hour fast.

The effect of TV in our and Khan's studies is probably caused by delayed gastric emptying, which postpones or prevents the resumption of the fasting pattern. These findings suggest that PGV does not delay gastric emptying as does TV, and that the proximal vagal supply to the stomach is unimportant in the control of fasting activity.

\section{References}

Allen, G. L., Poole, E. W., and Code, C. F. (1964). Relationships between electrical activities of antrum and duodenum. American Journal of Physiology, 207, 906-910.

Bedi, B. S., and Code, C. F. (1972). Pathway of coordination of postprandial, antral, and duodenal action potentials. American Journal of Physiology, 222, 1295-1298.

Code, C. F., and Marlett, J. A. (1975). The interdigestive myoelectric complex of the stomach and small bowel of dogs. Journal of Physiology, 246, 289-309.

Freund, J. E., (1973). Modern Elementary Statistics, p. 295. Prentice-Hall, International Inc.: London.

Hollander, F. (1948). Laboratory procedures in study of vagotomy. Gastroenterology, 11, 419-425.

Khan, I. H., and Bedi, B. S. (1972). Effect of vagotomy and pyloroplasty on the interdigestive myoelectrical complex of the stomach. Gut, 13, 841-842.

Marik, F., and Code, C. F. (1975). Control of the interdigestive myoelectric activity in dogs by the vagus nerves and pentagastrin. Gastroenterology, 69, 387-395.

Stoddard, C. J., and Duthie, H. L. (1973). The changes in gastroduodenal myoelectrical activity after varying degrees of vagal denervation. Gut, 14, 824.

Szurszewski, J. H. (1969). A migrating electric complex of the canine small intestine. American Journal of Physiology 217, 1757-1763. 
Thomas, J. E. (1957). Mechanics and regulation of gastric emptying. Physiological Reviews, 37, 453-474.

Thomas, J. E., and Crider, J. O. (1935). Rhythmic changes in duodenal motility associated with gastric peristalsis. American Journal of Physiology, 11, 124-129.

Weisbrodt, N. W., Copeland, E. M., Moore, E. P., Kearley, R. W., and Johnson, L. R. (1975). Effect of vagotomy on electrical activity of the small intestine of the dog. American Journal of Physiology, 228, 650-654.

Wilbur, B. G., and Kelly, K. A. (1973). Effect of proximal gastric complete gastric, and truncal vagotomy on canine gastric electric activity, motility and emptying. Annals of Surgery, 178, 295-303. 